\title{
Efficient Suppression of Endogenous CFTR Nonsense Mutations Using Anticodon Engineered Transfer RNAs
}

Wooree Ko ${ }^{1, \dagger}$, Joseph J. Porter ${ }^{1, \dagger}$, Matthew T. Sipple ${ }^{1}$, Katherine M. Edwards ${ }^{1}$, and John D. $\operatorname{Lueck}^{1,2,3, *}$

${ }^{1}$ Department of Pharmacology and Physiology, University of Rochester Medical School, Rochester, NY 14642, USA.

${ }^{2}$ Department of Neurology, University of Rochester Medical School, Rochester, NY 14642, USA.

${ }^{3}$ Center for RNA Biology, University of Rochester Medical School, Rochester, NY 14642, USA.

†Contributed equally

*Corresponding author. Email: john_lueck@urmc.rochester.edu

One Sentence Summary: Suppression of endogenous CFTR nonsense mutations by anticodon engineered tRNAs significantly increases mRNA expression and channel function.

Abstract: Nonsense mutations or premature termination codons (PTCs) comprise $\sim 11 \%$ of all genetic lesions, which result in over 7,000 distinct genetic diseases. Due to their outsized impact 
on human health, considerable effort has been made to find therapies for nonsense-associated diseases. Suppressor tRNAs have long been identified as a possible therapeutic for nonsenseassociated diseases, however their ability to inhibit nonsense-mediated mRNA decay (NMD) and support significant protein translation from endogenous transcripts has not been determined in mammalian cells. Here we investigated the ability of anticodon edited (ACE)-tRNAs to suppress cystic fibrosis (CF) causing PTCs in the cystic fibrosis transmembrane regulator $(C F T R)$ gene in gene-edited immortalized human bronchial epithelial (16HBEge) cells. Delivery of ACE-tRNAs to 16HBEge cells harboring three common CF mutations G542X-, R1162X- and W1282XCFTR PTCs significantly inhibited NMD and rescued endogenous mRNA expression.

Furthermore, delivery of our highly active leucine encoding ACE-tRNA resulted in rescue of W1282X-CFTR channel function to levels that significantly exceed the necessary CFTR channel function for therapeutic relevance. This study establishes the ACE-tRNA approach as a potential stand-alone therapeutic for nonsense-associated diseases due to its ability to rescue both mRNA and full-length protein expression from PTC containing endogenous genes.

\section{INTRODUCTION}

Nonsense mutations are single nucleotide mutations that convert a canonical amino acid codon to one of the three stop codons (UAA, UAG, UGA). Nonsense mutations result in a premature termination codon (PTC) generally with almost complete loss-of-function of the affected protein. This is due to both the PTC terminating translation prematurely, resulting in a truncated protein, and nonsense mediated mRNA decay (NMD) degrading the mRNA coding for the PTC-containing protein (1). With the advent of next-generation sequencing, more than 7,500 PTCs in nearly 1,000 different human genes have been discovered. PTCs account for close to 
$11 \%$ of all described mutations leading to inherited human disease (2). An abridged list of the known PTC-associated disease phenotypes includes Duchenne muscular dystrophy (3), spinal muscular atrophy (4), $\beta$-thalessemia (5), Hurler syndrome (6), Usher syndrome (7), and cystic fibrosis (8).

Cystic fibrosis (CF), a common autosomal recessive genetic disease, is caused by mutations to the cystic fibrosis transmembrane conductance regulator (CFTR) gene (9-11). Similar to genetic diseases as a whole, PTCs account for close to $10 \%$ of mutations causing CF (12). Several therapeutic small molecules rescue clinically relevant levels of CFTR function for a growing percentage of people with CF. However, all CF therapeutic small molecules so far target full-length aberrant CFTR protein (13-15). As PTCs result in little to no full-length protein, these small molecules targeting the CFTR protein are ineffective at rescuing PTC-containing CFTR. Instead, PTC therapies must target the defective gene, post-transcriptional processing, or processes governing protein translation.

Shortly after the discovery of the CFTR gene (9-11), proof-of-concept for gene replacement was demonstrated $(16,17)$. In the following 30 years, significant effort has been directed towards CFTR gene replacement therapy as this method has potential to repair the phenotypic defect for all people with CF. A number of viral and non-viral gene delivery approaches have been investigated (18). Improvements in vector design and delivery methods, a deeper understanding of lung biology and disease development, and new animal models have gotten us closer to the development of an effective mutation agnostic gene therapy for $\mathrm{CF}$, however to date no gene replacement therapy has made it to widespread clinical use (18).

CRISPR/Cas-based technologies including prime editing, homologous recombination following double-strand breaks, and in-frame deletions have received considerable interest as 
potential nonsense mutation interventions (19-21). As with total gene replacement, the CRISPR/Cas approach requires efficient delivery, optimally to progenitor cells, to ensure longterm repair of the nonsense mutation. Delivery of CRISPR/Cas both as an AAV encoding the CRISPR/Cas pair (20) and as a CRISPR/Cas ribonucleoprotein delivered with engineered amphophilic peptides (21) has been demonstrated in model systems. A drawback to CRISPR/Cas approaches is that design of a new guide RNA is required for each mutation site. As with gene therapy, an efficient delivery method for clinical use has not been determined.

Small molecules including aminoglycosides, synthetic aminoglycosides, and oxodiazoles have been reported to act as nonsense readthrough agents (22-24). Pharmacological treatment resulting in PTC readthrough is an attractive therapeutic avenue to pursue as these promise to allow for body-wide readthrough of PTCs. Small molecule PTC readthrough agents generally bind to the ribosome and reduce translational fidelity. While this allows for suppression of PTCs by near-cognate tRNAs, global translational fidelity is also decreased. Of note, several of these small molecule PTC readthrough agents have been investigated in clinical trials with generally low efficacy and strong oto- and nephrotoxicity (25). In addition, a recent study has also indicated that genome-wide natural termination codon (NTC) readthrough stimulated by the aminoglycoside G418 in vivo results in the disruption of several biological processes with detrimental cellular effects (26). While the promises of small molecule nonsense readthrough agents are tantalizing, due to issues with safety, efficacy, or both, their clinical implementation has remained out of reach.

Another nonsense suppression technology that has been explored previously is the use of nonsense suppressor tRNAs or anticodon edited tRNAs (ACE-tRNAs) (5, 27-31). A recently developed library of ACE-tRNAs with the anticodons engineered via mutagenesis to suppress 
one of the UAA, UAG, or UGA PTC codons demonstrated efficacious in vivo PTC suppression (30). While previous work by ourselves and others has demonstrated the safe and efficacious ability of ACE-tRNAs to rescue PTCs in the context of cDNAs, it is still unknown if they can take a major step forward in their therapeutic potential and rescue PTCs in a native genomic context. Heterologous overexpression of PTC-containing mRNAs as cDNAs has fundamentally limited relevance, as these constructs lack the genomic context/chromatin environment, native protein promoter, intronic sequences and post-transcriptional processing.

To that end, we set out to demonstrate ACE-tRNA based rescue of PTC-containing CFTR from the well characterized immortalized isogenic human bronchial epithelial (16HBE140-) cell lines modified with CRISPR/Cas9 to contain the most common CF-causing PTC variants (32). Here we show significant rescue of PTC-containing CFTR mRNA transcripts by real-time qRT-PCR for three of the most common CF-causing PTCs (G542X (2.5\%), W1282X (1.2\%), and R1162X (0.4\%)) (12) and significant rescue of CFTR protein function by patch clamp electrophysiology following delivery of our best performing ACE-tRNA ${ }^{\text {Leu }}$. The level of ACE-tRNA dependent rescue of functional W1282X-CFTR protein demonstrated here, is well above the predicted mark for therapeutically meaningful rescue $(33,34)$. While efficient delivery of ACE-tRNAs to affected cell types remains a hurdle to their therapeutic implementation, ACE-tRNAs display several attractive qualities as gene therapy agents bolstering their therapeutic promise (31). The results presented here display an exciting step forward in an unmet medical need for patients with nonsense mutations.

\section{RESULTS}

\section{Development of a nonsense suppression reporter system}


While nonsense mutations cause several serious genetic diseases, here we chose to focus on $\mathrm{CF}$, using the 16HBE14o- cell line and the derivative 16HBE gene edited (16HBEge) cell lines with engineered R1162X-, W1282X-, or G542X-CFTR variants (32). The genome of each of these 16HBEge cell lines was modified via CRISPR/Cas9 to contain a TGA stop codon at the amino acid residue specified in the CFTR gene. This model retains the native CFTR transcriptional, post-transcriptional, and translational regulation. We first set out to understand which previously identified nonsense suppressor ACE-tRNAs, whether delivered as DNA or RNA, were most effective. We also wanted to determine their persistence of activity and if previous results were recapitulated in human epithelial cells (30). To do this, we wanted a flexible high-throughput method to assess nonsense suppression in different cell lines, which could be transferred to any cell line of interest for future studies. Here we chose to use the piggyBac (PB) transposon/transposase system, which integrates the gene of interest (or cargo) specifically into genomic TTAA sites, facilitates fast selection of stable cell lines, can be used to generate both cell lines and animal models, and for which a hyperactive transposase has been generated (Fig. 1A) (35). We used a PTC-containing NanoLuc (NLuc) luciferase as a nonsense suppression reporter due to high signal-to-noise and utility in high-throughput assays(30). We sought an assay to determine the potentially short half-lives of ACE-tRNA therapeutic agents (i.e. delivered as RNA) for this we appended the protein-destabilizing PEST domain (Fig. 1B), which has been previously shown to shorten the half-life of NLuc to 20 minutes in several cell lines (36). Traditional NLuc assays require cell disruption as NLuc is retained intracellularly (36). By appending the IL6 secretion signal to the NLuc reporter (Fig. 1B), we developed a method to assay nonsense suppression longitudinally without disrupting cells. For longitudinal nonsense suppression studies the cell media is assayed for PTC-containing Sec-NLuc luminescence (Fig. 


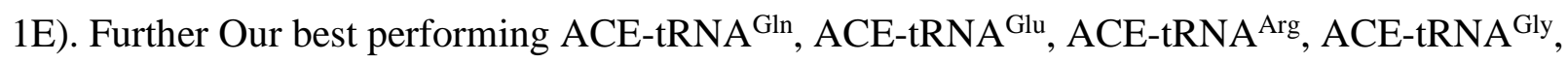
ACE-tRNA ${ }^{\text {Leu }}$, ACE-tRNA $^{\text {Trp }}$ library members displayed robust nonsense suppression for all three NLuc formats in 16HBE14o- cells, both as a transient co-transfection (Fig. 1C-E, Fig. S1AC) and following reporter stable integration (Fig. 1F, Fig. S1D). These results closely track what was displayed previously in HEK293T cells, indicating there is no overt cell-type-dependent impact on ACE-tRNA activity. 


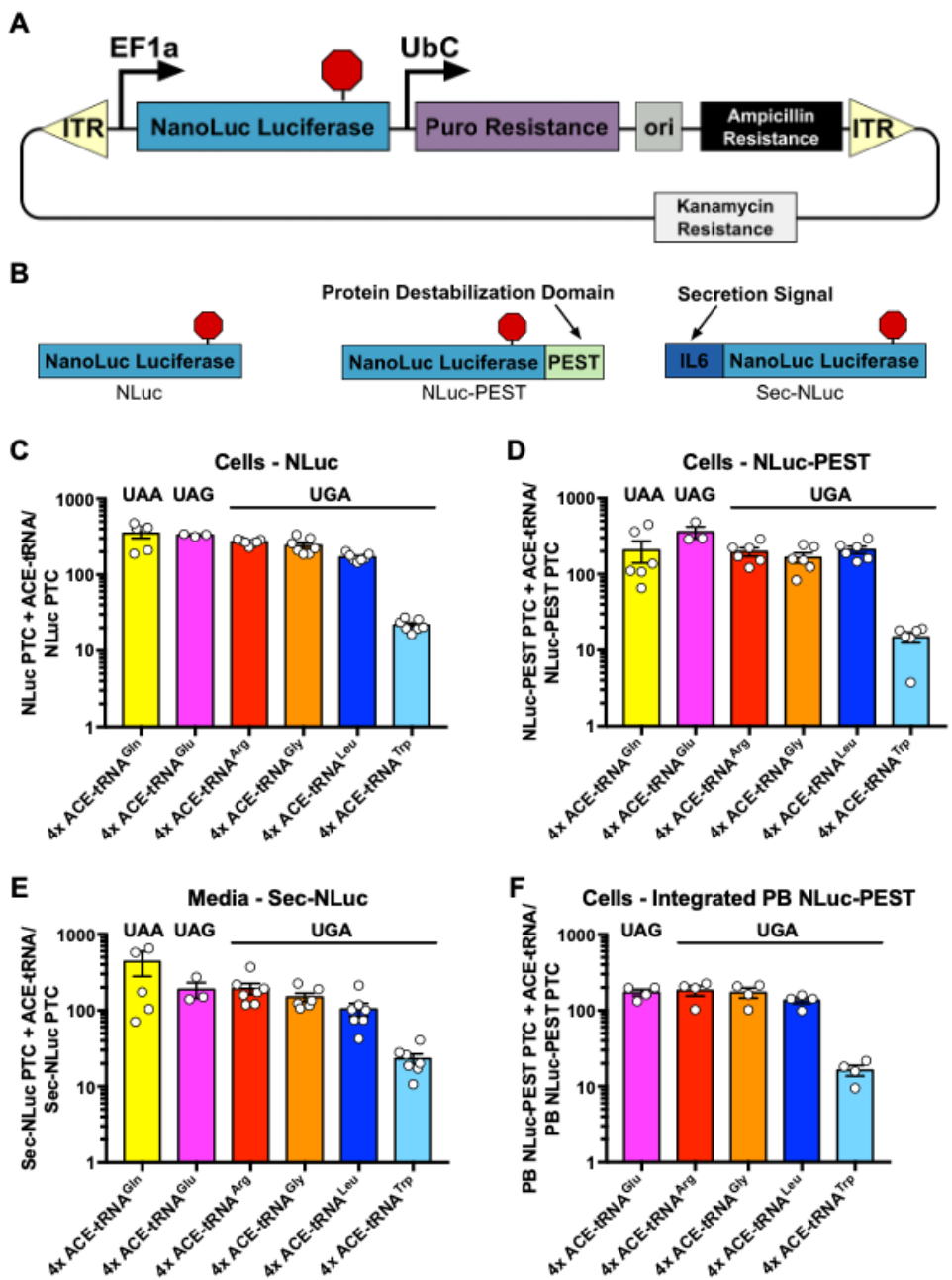

Figure 1. NLuc PTC reporter platforms faithfully report PTC suppression efficiency of ACE-tRNAs in 16HBE140- cells. (A) Schematic illustrates the piggyBac (PB) system used in this study. (B) Three types of NLuc PTC reporters were generated for reporting either UAA, UAG, or UGA PTC suppression efficiency of ACE-tRNAs: NLuc, NLuc with protein destabilization domain (NLuc-PEST) and NLuc with secretion signal (Sec-NLuc). The wildtype NLuc PTC reporter allows for high-throughput screening of different ACE-tRNAs, the NLuc-PEST PTC reporter with short intracellular lifetime allows for the measurement of the half-life of ACE-tRNA and the Sec-NLuc PTC reporter with secretion capacity allows for longitudinal studies. (C-F) In WT 16HBE14o- cells co-transfected with ACE-tRNAs and PTC reporters, six highest performing ACE-tRNAs suppressing UAA, UAG and UGA PTCs exhibit a similar trend of luminescence rescue for (C) NLuc, (D) NLuc-PEST and (E) Sec-NLuc reporters. (F) The NLuc-PEST PTC reporter stably integrated in WT 16HBE14o- cells using the PB system are capable of reporting PTC suppression efficiency of transiently transfected ACE-tRNAs through luminescence. Data are presented as normalized mean luminescence \pm SEM. $n=3-7$, with each replicate represented as a circle. Raw luminescence of described experiments is presented in Supplementary Fig. 1. 


\section{PTC suppression by ACE-tRNA encoded in plasmid peaks 48 hours following delivery to 16HBEge cells}

To assess the kinetics of nonsense suppression rescue, we used R1162X- and W1282X-CFTR 16HBEge cells stably integrated with PB transposon containing Sec-NLuc UGA. The cells were seeded on Transwells and transfected with a plasmid containing 4 copies of our top performing ACE-tRNA ${ }^{\text {Arg }}$, ACE-tRNA $^{\text {Trp }}$, and ACE-tRNA ${ }^{\text {Leu }}$ UGA suppressors, or an empty plasmid (control). Following transfection, the cells were seeded on Transwells and the Sec-NLuc luminescence was assayed daily from media collected from both the apical and basolateral sides of the Transwell. ACE-tRNA ${ }^{\text {Arg }}$ supported robust rescue of Sec-NLuc luminescence peaking around 2 days after transfection, in contrast to ACE-tRNA ${ }^{\text {Trp }}$, which performed relatively poorly (Fig. 2A-D). Indeed, the result with ACE-tRNA ${ }^{\text {Trp }}$ is not altogether unexpected, as it was the poorest performing ACE-tRNA in all of the most active ACE-tRNAs identified in our screen (30). The W1282L mutant of CFTR has been previously shown to support near-WT channel function (37), prompting us to test our highly performing ACE-tRNA ${ }^{\text {Leu }}$ for rescue of Sec-NLuc UGA in this assay. As with ACE-tRNA ${ }^{\text {Arg }}$, ACE-tRNA $^{\text {Leu }}$ supported robust rescue of Sec-NLuc luminescence peaking 2 days after transfection (Fig. 2A-D). Based on the results of this longitudinal study, it is likely that the decrease in luminescence rescue is based both on silencing and dilution of plasmids due to cell splitting $(38,39)$. Importantly, the luminescence-based assay informed us on when to perform more in-depth studies on CFTR mRNA expression and channel function described in this study. 


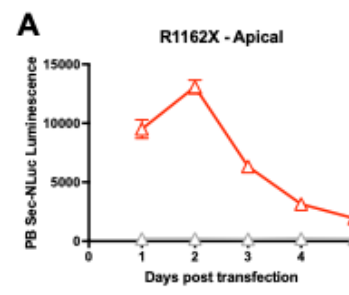

$\triangle \mathrm{PB}$ Seo-NLUE UGA+Empty

$\triangle$ PB SEO-NLUC UGA + 4XACE-ARNA

C

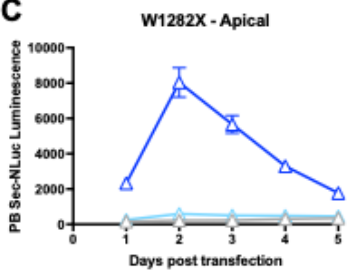

7.) PB Sec-NLuc UGA + Empty

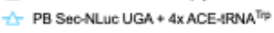

¿ PB SECNLUC UGA + 4XACE-APNALON

E

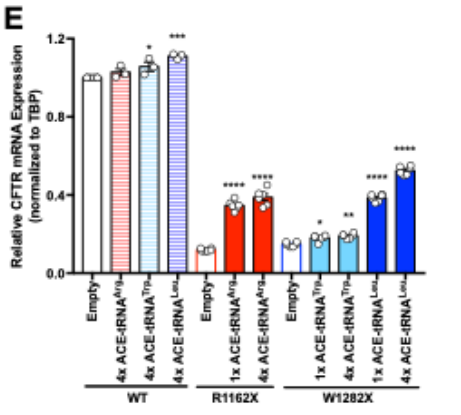

R1162X - Basolateral

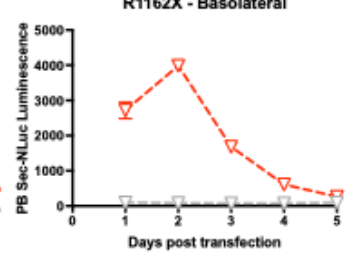

77. PB Sec-NLue UGA * Emply

$\Rightarrow$ PB Sec-NLUC UGA + 4XACE-ARNA AN

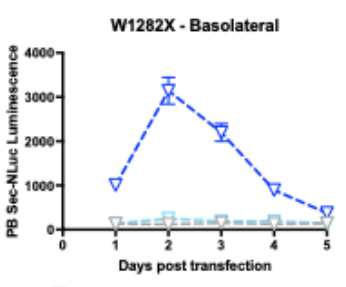

7. PB Sec-NLuc UGA + Emply

7. PB SeC-NLuc UGA + 4XACE-RRNATrip $\rightarrow$ PB Sec-NLue UGA + 4XACE-ARNA

F

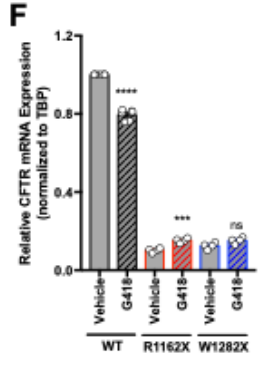

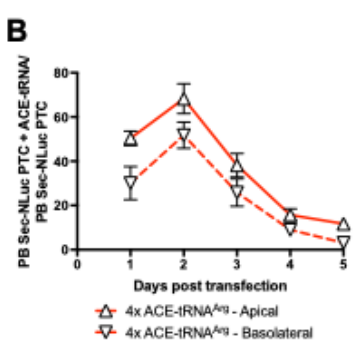

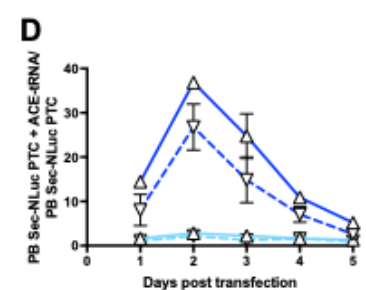

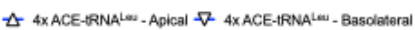
$\triangle 4 \times$ ACE-RRNATD - Apical $\nabla$ 4XACE-ARNATT - Basolateral

Figure 2. ACE-tRNAs delivered as plasmids have transient PTC suppression activity that significantly and robustly rescues endogenous $\mathrm{R} 1162 \mathrm{X}$ - and $\mathrm{W1282X}$-CFTR mRNA expression. (A-B) R1162X-CFTR and W1282X-CFTR cells stably expressing Sec-NLuc UGA reporter (R1162X PB Sec-NLuc UGA and W1282X PB Sec-NLuc UGA cells, respectively) were transfected with empty or 4x ACE-tRNA plasmids and luminescence measurements were made every $24 \mathrm{hrs}$ from of media 5 consecutive days. Transfection of (A, B) ACE-tRNA ${ }^{\text {Arg }}$ (solid red, $n=3$ ), (C, D) ACE-tRNA ${ }^{\text {Leu }}$ (dark blue, $n=3$ ) and (C, D) ACE-tRNA ${ }^{\text {Trp }}$ (light blue, $n=3$ ) plasmids results in time-dependent PTC suppression, with the peak luminescence detected at 2 days post transfection, in both the apical (solid lines) and basolateral (dashed lines) sides of Transwells. (A-C) Transfection of empty vectors (grey lines, $n=3$ ) results in negligible luminescence change over the course of 5 days. Data are shown in raw luminescence. (A, C) Raw luminesce and (B, D) luminescence normalized to cells receiving empty plasmids (ACEtRNA/Empty) display similar time-course of ACE-tRNA PTC suppression activity. (E) CFTR mRNA abundance normalized to TBP (housekeeping gene) in WT (white, $n=3$ ), R1162X-CFTR (red, $n=6$ ) and W1282X-CFTR (blue, $n=6$ ) cells $48 \mathrm{hrs}$ following transfection with empty (open) or 1x and 4x ACE-tRNA (filled) plasmids (E) Suppression of R1162X- and W1282X-CFTR, with ACE-tRNA ${ }^{\text {Arg }}$ and ACE-tRNA ${ }^{\text {Leu }}$ or ACE-tRNA ${ }^{\text {Trp }}$, respectively, significantly recovers the level of CFTR transcripts $48 \mathrm{hrs}$ after delivery as determined by real-time qRT-PCR. (F) Treatment with G418 $(100 \mu \mathrm{M})$, significantly increases CFTR mRNA expression in R1162X-CFTR cells (red) but not in W1282X-CFTR cells (blue). G418 treatment (gray, diagonal lines) significantly decreases CFTR mRNA expression in WT cells compared to vehicle treated cells (gray, open). Data are presented as average \pm SEM. Significance was determined by $(\mathbf{E})$ one-way ANOVA and Tukey's post-hoc test and (F) unpaired t-test, where $* P<0.05, * * P<0.01, * * * P<0.001$, and $* * * * P<0.0001$. 


\section{ACE-tRNAs rescue mRNA expression from endogenously encoded nonsense mutations}

The CFTR transcripts in R1162X-and W1282X-CFTR 16HBEge cells are sensitive to degradation through the NMD pathway (1). To that end, we determined the level of NMD inhibition elicited by transfection of ACE-tRNA plasmids. Total RNA was isolated from R1162X- and W1282X-CFTR 16HBEge cells 2 days after transfection of either 1-copy or 4copy ACE-tRNA ${ }^{\text {Arg }}$, ACE-tRNA ${ }^{\text {Trp }}$, ACE-tRNA $^{\text {Leu }}$ plasmids. We then performed real-time qRTPCR with probes against CFTR and control TATA-binding protein (TBP). ACE-tRNA ${ }^{\text {Arg }}$ and ACE-tRNA ${ }^{\text {Leu }}$, when delivered as plasmids containing 1-copy, rescued significant amounts of CFTR mRNA (34.6 \pm 1.0 and $38.5 \pm 0.8 \%$ of WT, respectively) as compared to cells transfected with an empty plasmid (11.9 \pm 0.3 and $14.8 \pm 0.4 \%$ of WT, respectively; Fig. 2E). Cells transfected with 4-copy ACE-tRNA plasmids also rescued significant amounts of CFTR mRNA (38.8 \pm 1.7 and $52.5 \pm 0.9 \%$ of WT, respectively), and to a greater extent than single copy ACEtRNA plasmids, suggesting multiple ACE-tRNAs within each delivered vector is beneficial in non-saturating conditions (Fig. 2E), which is consistent with previously reported results (40). ACE-tRNA dependent increase in CFTR mRNA expression is likely due to inhibition of NMDdependent mRNA degradation by suppressing the PTC during pioneer rounds of mRNA translation (1). In contrast to ACE-tRNA treatment, G418 (100uM) treatment significantly decreased WT CFTR mRNA abundance in $16 \mathrm{HBE} 14 \mathrm{o}$ - cells $(79.5 \pm 1.1 \%$ of vehicle treated 16HBE14o- cells), which is similar to a previously reported study (32). Reduction of WT CFTR mRNA expression following G418 treatment is likely due to global readthrough of NTCs and general translation miscoding (26), a feature that is not exhibited by ACE-tRNAs. Furthermore, treatment with G418 exhibited only a meager rescue of R1162X-CFTR mRNA expression (19.1 $\pm 0.5 \%$ of WT) compared to vehicle control (12.5 $\pm 0.8 \%$ of WT, Fig. $2 \mathrm{~F})$. To our knowledge, 
this is the first time that delivery of nonsense suppressor ACE-tRNAs has been directly shown to inhibit NMD and rescue mRNA from nonsense mutations in their endogenous genomic context.

\section{ACE-tRNA ${ }^{\text {Arg }}$ delivered as RNA suppresses nonsense mutations and rescues CFTR mRNA}

We next moved to ascertain whether ACE-tRNAs delivered as RNA could also suppress nonsense mutations in 16HBEge cells. As with plasmid delivery, we first wanted to determine the time-course of rescue in cells for ACE-tRNA as RNA delivered by nucleofection. Use of the stable PB Sec-NLuc UGA reporter 16HBEge cell line displayed significant rescue at day 1 but decreased sharply at day 2 (Fig. S2A-D). As we missed the initial increase in Sec-NLuc luminescence observed previously with cDNA delivery, we likely under-sampled and missed the peak PTC suppression time-point for RNA delivery. With that in mind, we shifted to the NLucPEST assay as NLuc with an appended PEST sequence has a half-life of about 20 minutes (36). The activity of ACE-tRNA ${ }^{\text {Arg }}$ as RNA peaks at $\sim 5$ hours and declines over the next 25 hours with a half-life of $\sim 6.5$ hours (Fig. 3A, Fig. S2E). Using this time-course as a guide, we assayed the ability of ACE-tRNA ${ }^{\text {Arg }}$ delivered as RNA to inhibit NMD at both 6 and 24 hours after nucleofection. We found R1162X-CFTR mRNA expression was significantly rescued by ACEtRNA $^{\text {Arg }}$ RNA $(31.9 \pm 3.7 \%$ of WT) compared to mock (no RNA) nucleofections $(13.0 \pm 0.6 \%$ of WT) at 6 hours post nucleofection, however this effect was not observed at 24 hours post nucleofection (Fig. 3B). ACE-tRNA delivered as RNA convincingly suppresses endogenous nonsense mutations, however to become a viable therapy we will likely need to extend the halflife of ACE-tRNA activity or perform frequent delivery. It also may be the case that the relatively short ACE-tRNA RNA half-life may be a product of the target cells, where an extended ACE-tRNA RNA may be observed in cells that are not actively undergoing mitosis (i.e 
airway epithelial cells in vivo). Transient delivery of ACE-tRNAs as both cDNA and RNA resulted in robust NMD inhibition at early time-points, unfortunately the time-course of PTC suppression activity did not allow us to measure ACE-tRNA dependent rescue CFTR channel activity using the Ussing chamber measurements because on average a minimum of 4 days is needed for formation of tight junctions (32). We therefore pursued the possibility of generating 16HBEge cells with stably integrated ACE-tRNA expression cassettes for persistent PTC activity. 

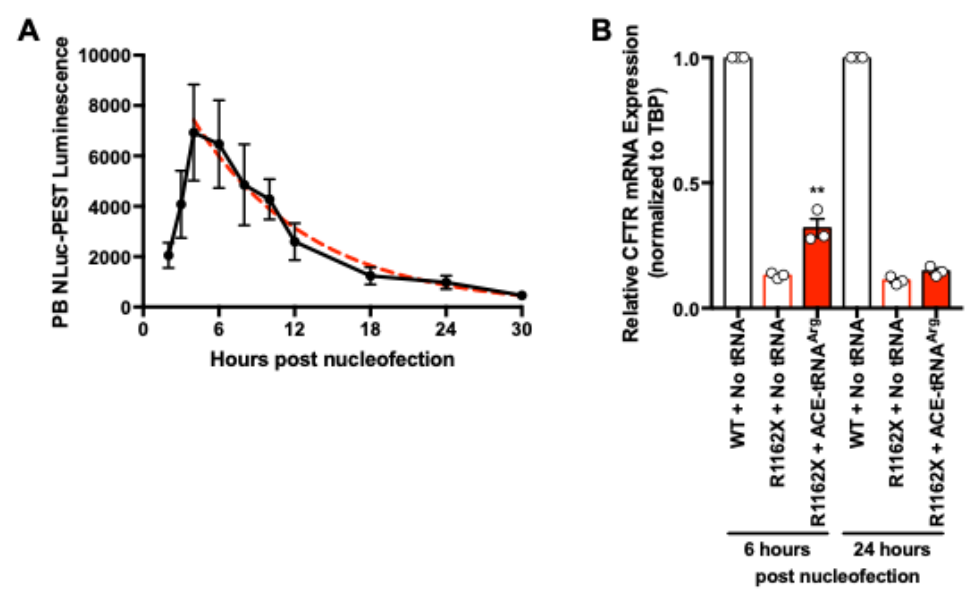

Figure 3. Delivery of ACE-tRNA ${ }^{\mathrm{Arg}}$ as RNA to R1162X-CFTR 16HBEge cells results in significant rescue of CFTR mRNA expression. (A) R1162X-CFTR cells stably expressing NLuc-PEST UGA reporter (R1162X PB PEST-NLuc UGA cells) were nucleofected with and without ACE-tRNA ${ }^{\operatorname{Arg}}$ RNA. Nonsense suppression mediated by ACE-tRNA ${ }^{\text {Arg }}$ was detected by luminescence measurements at 2, 3, 4, 6, 8, 10, 12, 18, 24 and 30 hours post nucleofection. Based on the average decay phase of luminescence by subtracting luminescence measurement with no delivered tRNA from that with ACE-tRNA ${ }^{\text {Arg }}$ RNA (red dotted line, $y=11413 \mathrm{e}^{-0.11 x}$ ), half-life of ACE-tRNA ${ }^{\operatorname{Arg}}$ is $6.5 \pm 0.3$ hours. Raw luminescence measurements are presented in Supplementary Fig. 2E. (B) After 6 hours (left) of delivery, ACE-tRNA ${ }^{\text {Arg }}$ (red filled) delivered as RNA in R1162X-CFTR cells is sufficient to significantly rescue R1162X-CFTR mRNA expression as determined by real-time qRT-PCR, from no RNA control (red open). No significant rescue of R1162X-CFTR mRNA expression was observed at 24hrs (right). Data are presented as average \pm SEM. All experiments contain an $n=3$. Significance was determined by unpaired t-test, where $* * P<0.01$. 


\section{Stable genomic integration of ACE-tRNA ${ }^{\text {Arg }}$ rescues significant amounts of CFTR mRNA and channel function in R1162X-CFTR 16HBEge cells}

Use of NLuc reporters allowed us to determine with ease a short time of action in both cDNA and RNA, likely due to rapid cell splitting of this model and dilution of ACE-tRNA plasmid. To circumvent this issue, we generated R1162X-CFTR 16HBEge cells containing stably integrated ACE-tRNA ${ }^{\text {Arg }}$ expression cassettes. For this purpose, we cloned 16 copies of ACE-tRNA ${ }^{\text {Arg }}$ along with a puromycin resistance marker into a PB transposon (Fig. S3A). R1162X-CFTR 16HBEge cells stably expressing PB ACE-tRNA ${ }^{\text {Arg }}$ were generated in a similar manner to the stable PB NLuc nonsense reporters described in Fig. 1 (see materials and methods). Of note, all studies with R1162X-CFTR 16HBEge cells stably integrated with 16 copies of ACE-tRNA ${ }^{\text {Arg }}$ transposon (R1162X PB 16x ACE-tRNA ${ }^{\text {Arg }}$ cells) were done from the total population of puromycin resistant cells which started from $>100$ colonies. We chose this approach to eliminate issues with genome insertion position effects of isogenic cell lines, which could pose issues with deconvoluting cell-to-cell variability. Given our ability to select 16HBEge cells stably expressing ACE-tRNA $^{\mathrm{Arg}}$, it assuages concerns that persistent expression of nonsense suppressor tRNAs at therapeutic levels will result in toxicity. Indeed, we observed that cells selected with puromycin exhibited similar morphology and cell division rates over $>20$ passages as compared to $16 \mathrm{HBEge}$ and 16HBE14o- cells, demonstrating that persistent ACE-tRNA expression is not toxic. To confirm that the stably incorporated ACE-tRNAs were functional, we transfected R1162X PB 16x ACE-tRNA ${ }^{\text {Arg }}$ cells with an NLuc UGA reporter construct. As expected, R1162X PB 16x ACE-tRNA Arg cells exhibited significant ( 600 fold) NLuc PTC suppression (Fig. 4A, filled red bar) as compared to stable R1162X-CFTR 16HBEge cells generated with a transposon 
bioRxiv preprint doi: https://doi.org/10.1101/2021.10.09.463783; this version posted October 9, 2021. The copyright holder for this preprint (which was not certified by peer review) is the author/funder. All rights reserved. No reuse allowed without permission.

containing puromycin resistance without ACE-tRNAs (R1162X PB Empty; Fig. 4A, open red bar). 


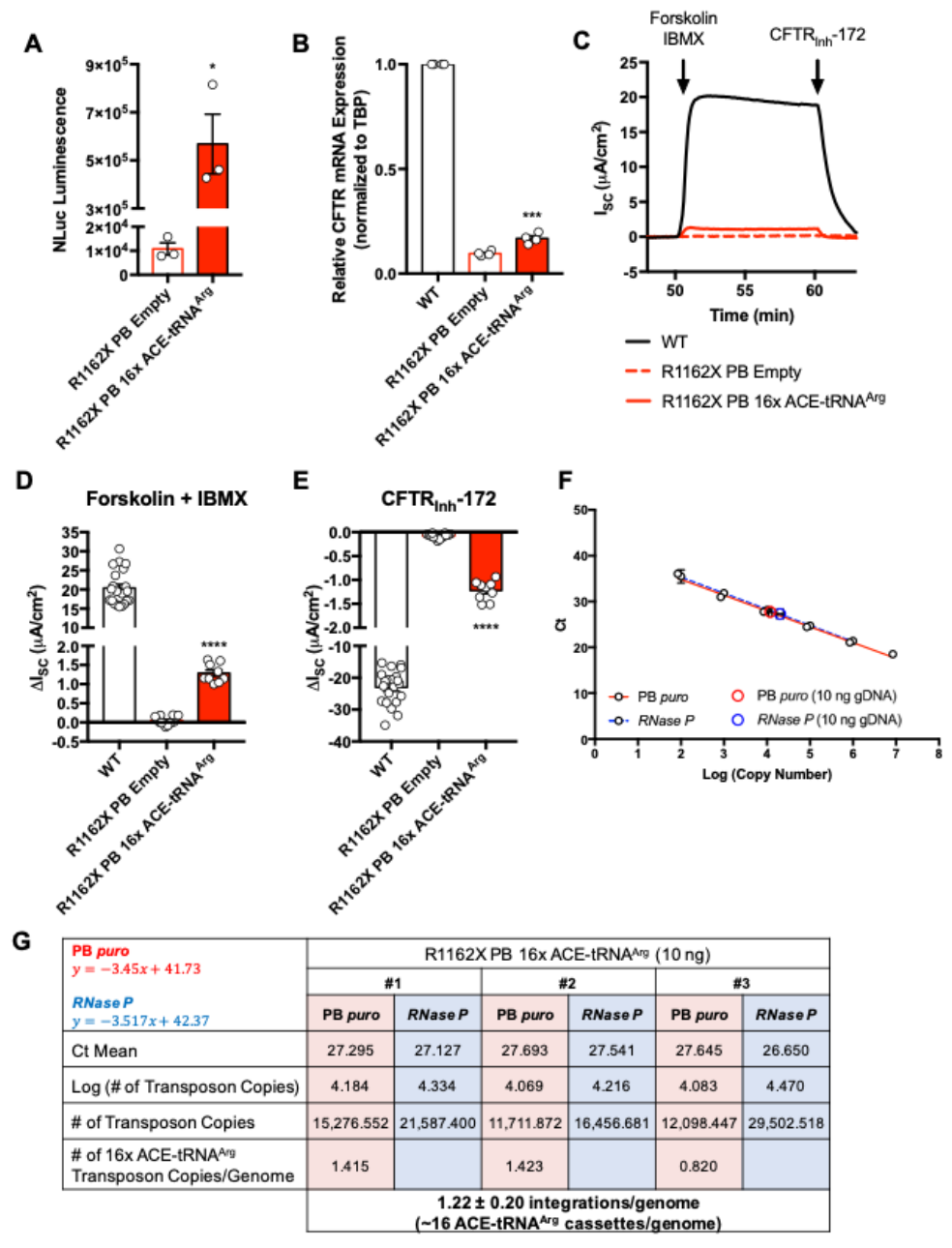

Figure 4. Stable integration of ACE-tRNA ${ }^{\text {Arg }}$ expression cassettes in R1162X-CFTR cells significantly rescues endogenous CFTR mRNA expression and CFTR channel function. (A) Raw luminescence measurement following transfection of the NLuc UGA reporter plasmid in R1162X-CFTR cells integrated with empty transposon (R1162X PB Empty; open red bar, $n=3$ ) and R1162X-CFTR cells stably integrated with 16x ACE-tRNA Arg transposon (R1162X PB 16x ACE-tRNA ${ }^{\mathrm{Arg}}$; filled red bar, $n=3$ ) results in significant suppression activity of integrated ACE-tRNA ${ }^{\text {Arg }}$. (B) R1162X-CFTR mRNA expression in R1162X PB 16x ACE-tRNA ${ }^{\text {Arg }}$ cells (filled red bar, $n=4)$ ) is significantly increased compared to R1162X PB Empty cells (open red bars, $n=4)$. (C)

Representative short-circuit $\mathrm{Cl}^{-}$current traces recorded from WT (black trace), R1162X PB Empty (dashed red trace) and R1162X PB 16x ACE-tRNA ${ }^{\text {Arg }}$ (solid red trace) cells in response to forskolin $(10 \mu \mathrm{M})$ and IBMX (100 $\mu \mathrm{M})$, followed by CFTR inhibitor, CFTR Inh- $^{-172}(20 \mu \mathrm{M})$. Average maximum ISC change in response to addition of

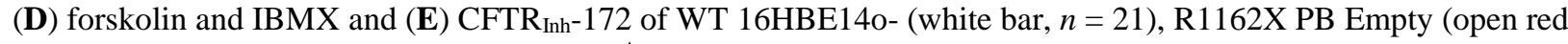
bar, $n=14$ ) and R1162X PB 16x ACE-tRNA ${ }^{\text {Arg }}$ (solid red bar, $\left.n=9\right)$ cells. (F) qPCR standard curves generated from 10-fold dilutions of PB 16x ACE-tRNA ${ }^{\mathrm{Arg}}$ (red line) and RNase P (Hs_RPP30 Positive Control; dashed blue) cDNA. qPCR measurements of PB puro transgene (open red circle) and endogenous RNase $P$ gene (open blue circle) from 10ng input of gDNA from R1162X PB 16x ACE-tRNA ${ }^{\text {Arg }}$ cells. Each sample was assayed in triplicate by qPCR. (G)16x ACE-tRNA ${ }^{\text {Arg }}$ transgene copy number per genome in R1162X PB 16x ACE-tRNA ${ }^{\text {Arg }}$ cells is calculated by plotting $C_{t}$ value to the standard curves. Data are presented as average $\pm S E M$. Significance was determined by unpaired t-test, where $* P<0.05$, $* * * P<0.001$, and $* * * * P<0.0001$. 
We performed real-time qRT-PCR of total RNA isolated from R1162X PB 16x ACE-tRNA ${ }^{\text {Arg }}$ cells noting a significant albeit modest $\sim 7 \%$ increase $(16.8 \pm 1.2 \%$ of WT) in CFTR mRNA as compared to mRNA from R1162X PB Empty cells $(9.6 \pm 0.5 \%$ of WT; Fig. 4B). In the face of this modest rescue of mRNA, we determined the extent of R1162X-CFTR functional channel rescue. To do so, we used Ussing chamber recordings of CFTR chloride short circuit currents (32). Representative traces display a forskolin and 3-isobutyl-1-methlyxanthine (IBMX) response over baseline and that this response is blocked by CFTRInh-172 (Fig. 4C), with average forskolin/IBMX and CFTR Inh-172 responses of CFTR channel function from R1162X PB 16x ACE-tRNA ${ }^{\text {Arg }}$ cells ranging from 5-7\% of WT CFTR channel function from 16HBE14o- cells (Fig. 4D). Using the R1162X PB 16x ACE-tRNA ${ }^{\text {Arg }}$ cells, we can now determine for the first time how many ACE-tRNA ${ }^{\text {Arg }}$ expression cassettes are needed to support significant rescue of CFTR mRNA expression and channel function. Previous studies demonstrated on average 1-3 PB transposon integrations per genome $(41,42)$. To count the average number of ACE-tRNA ${ }^{\text {Arg }}$ transposon integration events per cell, we used qPCR to compare signals from the integrated transposon to standard curves generated from known quantities of target cDNA. The RPP3O gene, subunit P30 of RNaseP enzyme complex, was used as a genomic DNA (gDNA) internal copy control, as this is a widely-accepted one copy gene of the haploid human genome (43). gDNA from R1162X PB 16x ACE-tRNA ${ }^{\text {Arg }}$ cells was isolated, and 10 ng was used as a template for real-time qPCR. The cycle threshold $\left(\mathrm{C}_{\mathrm{t}}\right)$ values obtained from primers and probes specific for $\mathrm{PB}$ puro (PB transposon puromycin resistance marker) and the RPP30 gene (RNase P) revealed that on average only $\sim 1$ copy of the PB transposon was integrated per genome, and therefore only 16 ACE-tRNA ${ }^{\mathrm{Arg}}$ expression cassettes are integrated per genome (Fig. 4F, G). Further experiments with $1 \mathrm{ng}$ of gDNA or a probe targeted against the PB backbone using either 
$10 \mathrm{ng}$ or $1 \mathrm{ng}$ of gDNA revealed a similar average number of integration events (Fig. S3B-D).

Indeed, we were excited to find that relatively few ACE-tRNA expression cassettes are required for significant rescue of both CFTR mRNA and channel function from endogenous CFTR genes with CF causing PTCs, which further provides promise for use of ACE-tRNAs as therapeutics for nonsense-associated $\mathrm{CF}$ as it reduces the burden of therapeutic delivery efficiency.

\section{ACE-tRNA expression yields significant rescue of CFTR mRNA expression in G542X-,}

\section{R1162X- and W1282X-CFTR 16HBEge cells.}

With the information that as few as 16 copies of ACE-tRNA ${ }^{\text {Arg }}$ support significant rescue of CFTR function, we revisited our experiments with transient transfection of plasmids encoding ACE-tRNAs, which do not recover more than $50 \%$ of the WT CFTR mRNA. Knowing that the transfection efficiency of $16 \mathrm{HBE} 14 \mathrm{o}-$ cells is $\sim 50 \%$, we wanted to determine if cDNA delivery is impacting our measurements of CFTR mRNA rescue. To account for transfection efficiency, we generated a transfection reporter plasmid containing both an EF1 $\alpha$-driven mNeonGreen $(\mathrm{mNG})$ and 4 copies of either ACE-tRNA ${ }^{\text {Gly }}$, ACE-tRNA $^{\mathrm{Arg}}, \mathrm{ACE}_{\mathrm{tRNA}}{ }^{\mathrm{Leu}}$, or ACE-tRNA ${ }^{\mathrm{Trp}}$

(Fig. 5A). mNG containing constructs permitted us to sort mNG positive (Fig. 5B, green curve) and negative (Fig. 5B, grey curves) cells by fluorescence-activated cell sorting (FACS) 48 hours after transfection and therefore determine ACE-tRNA dependent NMD inhibition in a nearhomogeneous population of cells that received ACE-tRNA encoding plasmids. FACS analysis determined the percentage of green cells, those receiving ACE-tRNA plasmid, in fact did not exceed 50\% (Fig. 5B, Fig. S4A, Supplemental Table 1). ACE-tRNA mNG positive G542X-, R1162X- and W1282X-CFTR 16HBEge cells exhibited significantly enhanced NMD inhibition over mNG negative cells (Fig. 5C). CFTR mRNA expression for ACE-tRNA ${ }^{\text {Gly_G542X was }}$ 


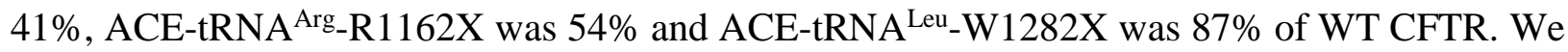
were excited to see that ACE-tRNA ${ }^{\text {Leu }}$ provided strong inhibition of NMD, indicating that when ACE-tRNAs are efficiently delivered to cells, they are exceedingly efficient at inhibiting NMD. The dependence of this result on transfection efficiency also highlights that ACE-tRNA delivery is a significant hurdle to their adoption as a therapeutic (see discussion). 

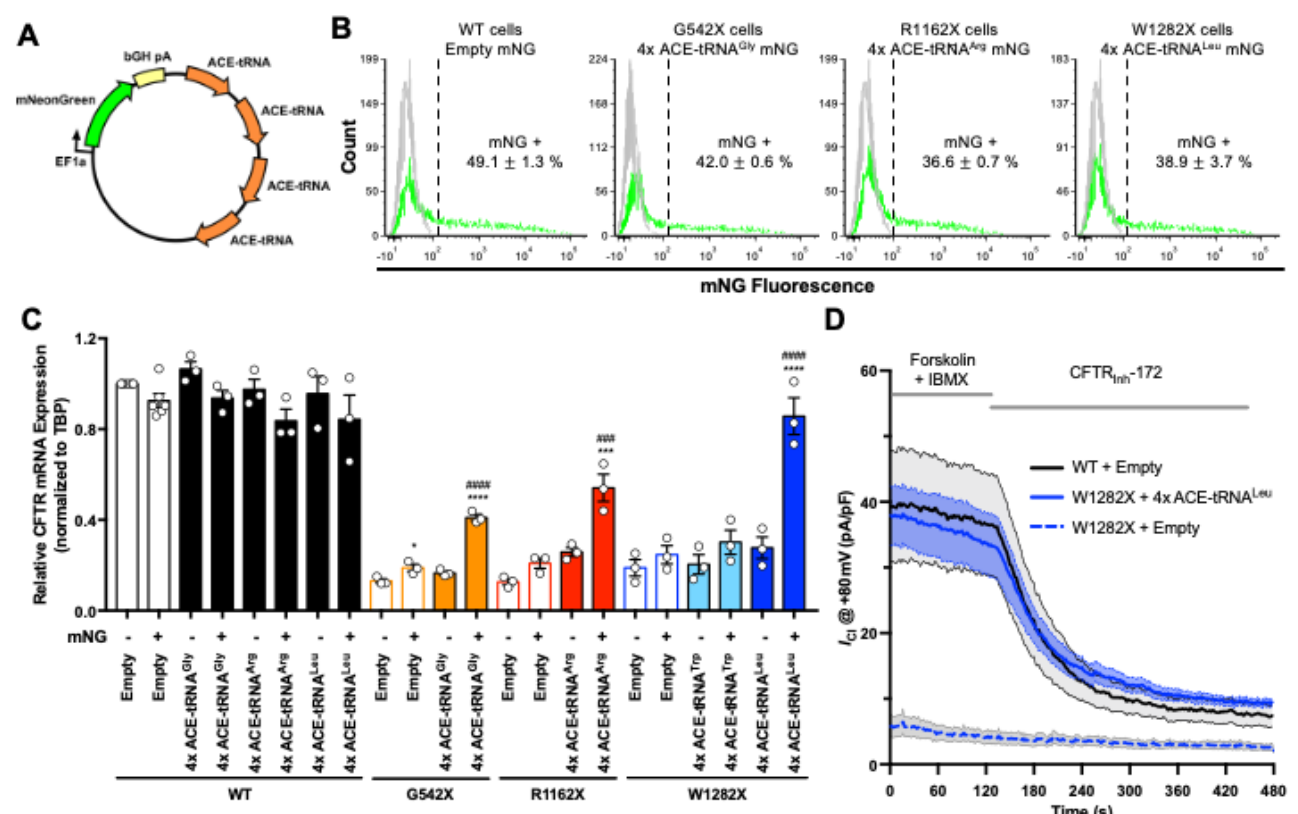

D

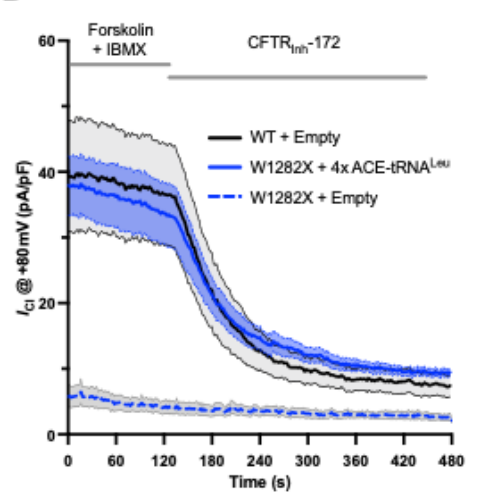

Figure 5. Expression of ACE-tRNAs results in significant and robust increase in CFTR mRNA and channel function. (A) Schematic of ACE-tRNA mNG plasmid. (B) $48 \mathrm{hrs}$ following transfection of WT, G542X-, R1162Xand W1282X-CFTR cells with plasmids encoding ACE-tRNAs and mNeonGreen (mNG) expression of fluorescent protein was determined by FACS to sort ACE-tRNA transfected cells (mNG positive) from non-transfected cells (mNG negative). Gray curves represent WT cells transfected with empty vectors, which were used to determine the gate (dashed line) for sorting mNG negative (ACE-tRNA not transfected) and positive (ACE-tRNA transfected, green curves) for each cell type and delivered plasmid. The percent $\mathrm{mNG}$ positive cell population is indicated in each panel. Representative FACS histograms for WT 16HBE14o- cells (top) and paired empty plasmid transfection of G542X-, W1282X- and R1162X-CFTR cells (bottom) are presented in Supplemental Fig. 4A. Representative FACS histograms of cells transfected with control empty plasmids are presented in Supplemental Fig. 4B. (C) Real-time qRT-PCR quantification of WT, G542X-, R1162X- and W1282X-CFTR mRNA expression in FACS sorted $\mathrm{mNG}$ positive and negative cell populations following transfection of $\mathrm{mNG}$ plasmids encoding ACE-tRNA ${ }^{\mathrm{Gly}}$ (orange bars), ACE-tRNA ${ }^{\text {Arg }}$ (red), ACE-tRNA ${ }^{\text {Trp }}$ (light blue bars) and ACE-tRNA ${ }^{\text {Leu }}$ (dark blue bars). mNG positive and negative WT 16HBE14o- cells transfected with empty (white bars, $n=4$ ) and ACE-tRNA ${ }^{\text {Gly }}$ (black bars, $n=3$ ), ACE-tRNA Arg (black bars, $n=3$ ), ACE-tRNA ${ }^{\text {Trp }}$ (black bars, $n=3$ ), and ACE-tRNA ${ }^{\text {Leu }}$ (black bars, $n=$ 3), did not exhibit significantly altered CFTR mRNA expression. Transfection of empty mNG plasmids into R1162X- (open red bars, $n=3$ ) and W1282X-CFTR (open blue bars, $n=3$ ) cells did not significantly affect CFTR mRNA expression, while mNG positive G542X-CFTR cells (open orange bars, $n=3$ ) exhibited a significant but meager increase in CFTR mRNA expression. mNG positive G542X-, R1162X- and W1282X-CFTR cell populations exhibited a significant and robust rescue of CFTR mRNA expression over $\mathrm{mNG}$ negative cell populations 48hrs following transfection with 4x ACE-tRNA ${ }^{\text {Gly }}$ (solid orange bars, $n=3$ ), ACE-tRNA ${ }^{\text {Arg }}$ (solid red

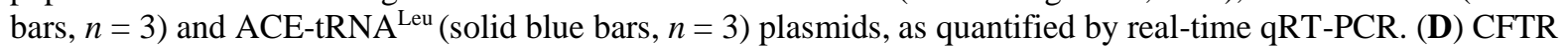
channel activity was assessed in control empty mNG transfected WT 16HBE14o- cells (black trace, $n=7$ ), W1282X-CFTR cells (dashed blue trace, $n=5$ ), and 4x ACE-tRNA ${ }^{\text {Leu }}$ mNG plasmid transfected W1282X-CFTR cells (blue trace, $n=11$ ) using whole-cell voltage clamp. Results in $\mathbf{C}$ and $\mathbf{D}$ are presented as average \pm SEM, and significance was determined by one-way ANOVA and Tukey's post-hoc test, where $* P<0.5$ vs. mNG negative, **** $P<0.0001$ vs. $\mathrm{mNG}$ negative, ${ }^{\# \# \#} P<0.001$ vs. empty $\mathrm{mNG}$ positive and ${ }^{\# \# \# \#} P<0.0001$ vs. empty $\mathrm{mNG}$ positive. 


\section{Whole-cell patch clamp reveals near-WT rescue of CFTR channel function from endogenous nonsense mutations with ACE-tRNAs}

Although we demonstrated significant and robust rescue of CFTR mRNA in G542X-, R1162Xand W1282X-CFTR 16HBEge cells, a rescue of 15-30\% of CFTR channel function is ultimately required for a therapeutically relevant outcome (34). To this end, we transfected 4x ACEtRNA $^{\text {Leu }}$ mNG plasmids (Fig. 5A) into W1282X-CFTR 16HBEge cells and utilized the wholecell patch clamp technique to directly measure CFTR channel function in mNG positive cells 4872 hours after transfection (Fig. 5D). To fully activate CFTR channels, we pre-incubated cells in forskolin $(20 \mu \mathrm{M})$ and IBMX $(100 \mu \mathrm{M})$ for 5 minutes before starting the recording. We propose that measuring CFTR function under these conditions provides a theoretical upper limit to ACEtRNA dependent PTC suppression efficiency of endogenous CFTR. Here, ACE-tRNA ${ }^{\text {Leu }}$ mNG positive W1282X-CFTR 16HBEge cells exhibited CFTRInh-172 sensitive chloride current magnitudes (Fig. 5D, blue trace, $26.7 \pm 4.3 \mathrm{pA} / \mathrm{pF}$ at $+80 \mathrm{mV}, \mathrm{n}=11$ ) that were $91 \%$ of those measured in WT 16HBE14o- cells (Fig. 5D, black trace, $29.3 \pm 6.2 \mathrm{pA} / \mathrm{pF}$ at $+80 \mathrm{mV}, \mathrm{n}=7$ ). As expected, W1282X-CFTR 16HBEge cells receiving empty mNG plasmids exhibited no significant CFTR function (Fig. 5D, dashed blue trace, $n=5$ ). These results are consistent with Ussing chamber measurements of R1162X PB 16x ACE-tRNA Arg cells (Fig. 4B), where CFTR channel function appears to closely track CFTR mRNA expression.

\section{DISCUSSION}

Several studies have used nonsense suppressor tRNAs to address PTCs in certain disease-related genes including $\beta$-globin in Xenopus laevis oocytes (27), xeroderma pigmentosum group A in human cells (28), chloramphenicol acetyl transferase in transgenic mice (40), CDH1 in human 
cell lines (29), and CFTR in human cells and Xenopus laevis oocytes (30). We previously demonstrated using mass spectrometry of the full-length protein product of PTC readthrough that ACE-tRNAs in their current form insert the correct amino acid, providing seamless rescue of the PTC (30). While these studies indicated that ACE-tRNAs are competent to promote suppression of PTCs in mRNA produced from cDNA, they do not fully recapitulate the hurdles seen in the rescue of genuine PTCs in their genomic context. NMD is a eukaryotic translation-dependent mRNA quality control pathway that selectively degrades mRNA transcripts containing PTCs (1, 44). Here we demonstrate CFTR mRNA rescue from 16HBEge cells containing PTCs in the endogenous genomic context. Electrophysiological assays including Ussing chamber short circuit current recording and patch clamp are considered the 'gold standard' for evaluating CFTR functional rescue in drug development (32). Using these assays, we demonstrate protein rescue of PTC-containing CFTR, providing proof-of-principle that ACE-tRNAs when delivered as cDNA or RNA can rescue native PTCs. Based on our work here, we note that protein rescue generally tracks with mRNA rescue from NMD. This work establishes a theoretical upper limit to the therapeutic window as $>90 \%$ of WT function recovered when selecting for efficient delivery of ACE-tRNA as DNA.

While this study represents PTC rescue in one of the most faithful PTC models used to date when assaying for function of ACE-tRNAs, a fundamental limitation is that this is an immortalized cell line and does not fully recapitulate the delivery issues or potential safety issues realized in vivo. A notable concern about the safety of ACE-tRNA is that nonsense suppressor tRNAs may cause readthrough of NTCs to toxic effect. In our previous work, we determined via ribosome profiling that readthrough of NTCs is minimal in comparison to PTCs (30). Further, in the context of genetic code expansion, stable expression, and viral delivery of nonsense 
suppressor tRNAs has been shown to be tolerated in mice $(45,46)$. Our ability to generate cell lines stably expressing 16 copies of ACE-tRNAs with similar growth rates and morphology to WT parents cells lends further credibility to tolerably low levels of toxicity due to NTC readthrough. It should also be noted that ACE-tRNAs inherently possess a lower level of global NTC readthrough. Unlike the majority of small molecule PTC readthrough agents developed to date, ACE-tRNAs only suppress one of the three stop codons. A further advantage of ACEtRNAs over small molecule PTC readthrough agents highlighted here is that ACE-tRNAs provide a la carte rescue, that is the amino acid encoded at the PTC can be specified (i.e. Leu at W1282X). While results shown by ourselves and others indicate the safety of ACE-tRNAs, the DNA delivery methods used here require the use of a transfection marker to control for efficient DNA delivery and cannot be directly translated to the clinic. This highlights a remaining roadblock of delivery to the clinical implementation of ACE-tRNAs, however it should be noted that tRNAs as gene therapy cargo possess a number of attractive qualities (31). We have shown here that ACE-tRNAs can be delivered as RNA or DNA, for which there are many therapeutically relevant delivery avenues to pursue.

As demonstrated here, ACE-tRNAs delivered as RNA are competent to rescue the expression of endogenous PTC-containing R1162X-CFTR mRNA. Given that CFTR protein rescue generally tracks with mRNA rescue, it follows that ACE-tRNA delivered as RNA will rescue protein from genes containing endogenous PTCs. However, due to technical limitations of the model cell line used here, we have not demonstrated CFTR protein rescue directly using ACE-tRNA as RNA. While ACE-tRNA delivered as RNA can go through multiple rounds of aminoacylation and translation, it is not a renewable PTC suppression resource like ACE-tRNA delivered as DNA. Therefore, it is likely that repeated delivery of ACE-tRNA would be needed to sustain a 
therapeutic level of target protein expression and that modifications to the RNA may be necessary to extend the half-life of the ACE-tRNA therapeutic agent. However, because ACEtRNAs are only $~ 76$ nucleotides in length, many of the existing mRNA and short RNA delivery technologies can likely be adapted for delivery of ACE-tRNAs. These delivery methods include but are not limited to cationic or ionizable lipids and lipid-like materials and polymers/nanoparticles (47). In contrast to ACE-tRNA delivered as RNA, delivery as DNA likely will result in augmented persistence of activity.

When ACE-tRNAs are efficiently delivered as DNA, we demonstrate here rescue of both PTCcontaining CFTR mRNA and near $100 \%$ rescue of CFTR protein, far exceeding the therapeutic threshold for functional CFTR rescue of $15-30 \%$ (34). Due to the small size of ACE-tRNA expression cassette ( 250 nucleotides), there are a number of avenues available to deliver ACEtRNA DNA expression cassettes. The small size of the ACE-tRNA expression cassette allows for optimization of cassette copy number with a minimal vector size which in turn has been shown to increase intracellular mobility, nuclear entry, and gene therapy agent expression (4850). Naked DNA delivery is an attractive gene therapy approach as it has inherently low immunogenicity, can be designed to remain episomal or integrate, and can be paired with several technologies including nanoparticle formulations, liposomes, pressure injection, and electroporation (51-53). When plasmid DNA is made resistant to silencing, several studies have shown extended expression (months) in murine lung following plasmid DNA delivery by polyethyleneimine/DNA complexes (54), cationic liposome/DNA complexes (55), and DNA electroporation (56).

The small expression cassette size of ACE-tRNAs also make them amenable to delivery by a variety of viral vectors. Viral delivery is an attractive gene therapy approach as viral cargo 
delivery is usually much more efficient than non-viral delivery and has the advantage of celltype-specific transduction. There are a number of candidate viral vectors, some of which include adenovirus, adeno-associated virus (AAV), and lentivirus (57). Recently the FDA approved the use of the first AAV therapeutic for total gene replacement therapy of RPE65 (retinal pigment epithelial-specific $65 \mathrm{kDa}$ protein) for treatment of the inherited retinal disorder Leber congenital amaurosis type-2 (Luxturna $\left.{ }^{\circledR}\right)$. This demonstrates the possibility of therapeutic a possible route to viral delivery of ACE-tRNAs. Of note, ACE-tRNAs are particularly amenable to AAVs as their small size allows for multiple expression cassettes despite the relatively small cargo capacity of AAVs $(<4.7 \mathrm{~kb})$. Indeed, for many diseases associated with nonsense mutations, AAV total gene replacement is not viable due to the large size of the gene in question. In contrast, ACE-tRNA-mediated PTC suppression is independent of target gene size, meaning ACE-tRNAs delivered as an AAV can rescue gene targets of any size. As we demonstrated here that integrating up to 16 copies of ACE-tRNA ${ }^{\text {Arg }}$ was well tolerated in 16HBEge cells, the use of integrating lentiviral vectors for one-time delivery of ACE-tRNAs is another attractive approach.

In summary, nonsense mutations cause $\sim 11 \%$ of all serious genetic diseases including $\mathrm{CF}$, for which there are currently few therapeutic options even given their unitary mechanism. Our results suggest that nonsense suppressor ACE-tRNAs are capable of rescuing both PTCcontaining mRNA from NMD and functional protein expression. Coupled with an efficient RNA or DNA delivery technology, ACE-tRNAs may provide relief for a variety of genetic diseases in the future.

\section{MATERIALS AND METHODS}




\section{Materials for Molecular Biology}

Unless otherwise specified, all molecular biology reagents were obtained from New England Biolabs. For all PCR reactions Q5 High-Fidelity 2X Master Mix (NEB M0492L) was used, Gibson assembly was performed using NEBuilder HiFi DNA Assembly Master Mix (NEB E2621L), Golden Gate reactions used T4 DNA ligase (NEB M0202L) and restriction enzymes from NEB, gel purifications were performed using the Monarch DNA Gel Extraction Kit (NEB T1020L), and minipreps were performed using the Monarch Plasmid Miniprep Kit (NEB T1010L). NEB 5-alpha Competent E. coli (NEB C2987H) were used for all bacterial transformations unless otherwise noted. All oligonucleotides were synthesized by Integrated DNA Technologies. All cDNA preparations were performed using the NucleoBond Xtra Midi EF kit (Macherey Nagel Ref 740420.50).

\section{DNA constructs/Plasmid generation}

The piggyBac transposon plasmid (PB plasmid) with piggyBac inverted terminal repeats (ITRs) flanking an ampicillin resistance marker, pBR322 origin, short ubiquitin C promoter (UbC)puromycin $N$-acetyltransferase-SV40 pA, and EcoRV, PmII, PmeI, SmaI multiple cloning site in pUC57 was synthesized (Genscript). The hyperPB transposase sequence (58) was synthesized as gBlocks (IDT, Coralville, IA, USA) and Gibson Assembled into pcDNA3.1(+). For further specifics of plasmid cloning methods see supplemental materials and methods.

\section{Cell culture}

Parental 16HBE140- human bronchial epithelial cells, which were generated by Dieter Gruenert (University of California, San Francisco) (59), and 16HBEge cells with engineered CFTR 
variants, CFF-16HBEge CFTR G542X, CFF-16HBEge CFTR R1162X and CFF-16HBEge

CFTR W1282X cells, were obtained from the Cystic Fibrosis Foundation Therapeutics Lab (Lexington, MA, USA) (32). HBE cells were cultured in Minimum Essential Medium (MEM; Gibco, \#11095-080) supplemented with 10\% Fetal Bovine Serum (Gibco, \#26140-079) and 1\% Penicillin/Streptomycin/Glutamine (Gibco, \#10378-016) in a $37{ }^{\circ} \mathrm{C} / 5 \% \mathrm{CO}_{2}$ humidified incubator. Plates were coated with coating solution [LHC-8 basal medium (Gibco, \#12677-027), $1.34 \mu \mathrm{l} / \mathrm{ml}$ Bovine serum albumin 7.5\% (Gibco, \#15260-037), $10 \mu 1 / \mathrm{ml}$ Bovine collagen solution, Type 1 (Advanced BioMatrix, \#5005-100ML) and $10 \mu 1 / \mathrm{ml}$ Fibronectin from human plasma (Thermo Fisher Scientific, \#33016-015)] for 2-3 h at $37{ }^{\circ} \mathrm{C} / 5 \% \mathrm{CO}_{2}$ and stored at $4{ }^{\circ} \mathrm{C}$ after thorough removal of coating solution.

\section{Transfection and Nucleofection of HBE cells}

HBE cells were transfected with Lipofectamine LTX and Plus reagent (Invitrogen by Thermo Fisher Scientific, \#15338-100) according to manufacturer's guidelines with slight modifications. Briefly, on the day of transfection, 500,000 cells per well were plated in a 6-well plate and incubated in a cell culture incubator for 20-30 minutes. $2.5 \mu \mathrm{g}$ of DNA and 2.5 $\mu \mathrm{l}$ of PLUS reagent were diluted in $125 \mu$ l of Opti-MEM (Gibco, \#51985-034), and $5 \mu 1$ of Lipofectamine LTX reagent was diluted in Opti-MEM. Diluted cDNA and Lipofectamine LTX reagent were combined, mixed thoroughly and incubated for 5 minutes at room temperature. The transfection mixture was then added dropwise to pre-plated cells. The media containing transfection mix was replaced with normal cell culture media 16-24 hours post transfection. HBE cells were nucleofected with the Amaxa 4D-Nucleofector system (Lonza, Basel, Switzerland) using the Amaxa SG cell line 4D-Nucleofector X Kit S (Lonza, \#V4XC-3032). Each well of a black 96- 
well plate was filled with $95 \mu \mathrm{l}$ of cell culture media and pre-incubated in a humidified $37{ }^{\circ} \mathrm{C} / 5 \%$ $\mathrm{CO}_{2}$ incubator, and an aliquot of cell culture media was pre-warmed in a $37^{\circ} \mathrm{C}$ water bath. For each reaction, 200,000 cells were centrifuged at $90 \mathrm{x}$ g for 10 minutes, resuspended in $20 \mu \mathrm{l}$ of supplemented SG 4D-Nucleofector X solution and mixed with $1 \mu \mathrm{g}$ of RNA (maximum 10\% of final sample volume). A total volume of $20 \mu \mathrm{l}$ was transferred to a well of Nucleocuvette Strip. The nucleofection process was started by placing the strip into the 4D-Nucleofector X Unit and running the CM-137 Nucleofector program from the 4D-Nucleofector Core Unit. Cells were incubated at room temperature for 10 minutes and resuspended with $80 \mu$ l of pre-warmed cell culture media. $15 \mu \mathrm{l}$ of nucleofected cells were plated in pre-incubated cell culture plate and maintained in a humidified $37^{\circ} \mathrm{C} / 5 \% \mathrm{CO}_{2}$ incubator until analysis.

\section{Stable cell line generation}

To generate stable HBE cell lines, the piggyBac $(\mathrm{PB})$ transposon vector expressing gene of interest (NLuc PTC reporter or ACE-tRNA) was co-transfected with the PB transposase vector. For each well of a 12-well plate, 250,000 cells were seeded on the day of transfection and transfected with $0.5 \mu \mathrm{g}$ of $\mathrm{PB}$ transposon vector and $0.5 \mu \mathrm{g}$ of empty vector as a negative control or $0.5 \mu \mathrm{g}$ of PB transposon vector and $0.5 \mu \mathrm{g}$ of PB transposase vector using Lipofectamine LTX and Plus reagent as described above. Stable cells were selected with $0.5 \mu \mathrm{g} / \mathrm{ml}$ of puromycin (InvivoGen, \#ant-pr-1) 1-2 days post transfection until no viable cells were detected in the negative control condition.

\section{Fluorescence-activated cell sorting (FACS)}


For fluorescence-activated cell sorting (FACS) experiment, cells were washed with Dulbecco's phosphate-buffered saline (DPBS; Gibco, \#14190-144) twice, dissociated with TrypLE Express (Gibco, \#12604-013), pelleted at $1000 \mathrm{rpm}(\sim 500 \mathrm{xg})$ for 5 minutes, resuspended with DPBS with calcium and magnesium (Gibco, \#14040-133) and strained with $40 \mu \mathrm{m}$ Cell Strainer. Cells were then sorted using the BD FACSAria II cell sorter (BD Biosciences), and data were analyzed by using FCS Express 7 flow cytometry software (De Novo Software, Pasadena, CA, USA).

In order to sort ACE-tRNA expressing cells from ACE-tRNA not expressing cells, HBE cells were transfected with the plasmid containing ACE-tRNA and mNeonGreen (mNG) fluorescent protein in one plasmid. Approximately 36-48 hours after transfection, cells were prepared as described above for FACS experiment. Empty vector expressing cells were used to determine the gate for mNG negative and positive cells. Each population of $\mathrm{mNG}$ negative and positive cells was then sorted into a $15 \mathrm{ml}$ conical tube using the BD FACSAria II cell sorter. Total RNA was isolated from sorted cells and used for RT-qPCR as described below.

\section{Luciferase reporter assay}

PTC readthrough by ACE-tRNA was assessed by measuring NanoLuc (NLuc) luciferase activity using the Nano-Glo Luciferase Assay System (Promega, \#N1130) on a Synergy2 multi-mode microplate reader (BioTek Instruments, Winooski, VT, USA). Using Lipofectamine LTX and Plus reagent as described above, HBE cells were co-transfected with NLuc, NLuc-PEST or SecNLuc PTC reporters and ACE-tRNA, or HBE cells stably expressing PTC reporters were transfected with ACE-tRNA. In each well of a black 96-well plate, $15 \mu 1$ of DPBS was added for cells expressing NLuc and NLuc-PEST PTC reporters, whereas $15 \mu \mathrm{l}$ of media from transfected 
cells were transferred for cells expressing Sec-NLuc PTC reporter. Then, $15 \mu 1$ of reconstituted Nano-Glo Luciferase Assay Reagent (1:50 of Nano-Glo Luciferase Assay Substrate to Nano-Glo Luciferase Assay Buffer) was added to each well for luminescence recording using a Synergy2 multi-mode microplate reader. Each condition was assayed in triplicate, and cells expressing empty vector instead of ACE-tRNA were included as a negative control. Luciferase activity was displayed as raw NLuc luminescence or normalized NLuc luminescence to background luminescence.

\section{Quantitative reverse transcription polymerase chain reaction (RT-qPCR)}

Total RNA was isolated from cells with the Monarch Total RNA Miniprep Kit (New England BioLabs, \#T2010S) according to manufacturer's recommendations. RNA quantity and quality were measured with a NanoDrop One ${ }^{c}$ Spectrophotometer (Thermo Fisher Scientific, Waltham, MA, USA). Subsequently, one-step reverse transcriptase and quantitative PCR (RT-qPCR) was performed on the QuantStudio 3 Real-Time PCR System (Applied Biosystems, Waltham, MA, USA) using the Luna Universal One-Step RT-qPCR Kit (New England BioLabs, \#E3005L or \#E3006E) according to manufacturer's instructions and then analyzed with QuantStudio Design \& Analysis Software v1.5.1 (Applied Biosystems, Waltham, MA, USA). Briefly, the reaction mixture contained $10 \mu 1$ 2x Luna Universal Probe One-Step Reaction Mix, $1 \mu 1$ 20x Luna WarmStart RT Enzyme Mix, $1 \mu 1$ 20x CFTR-specific primers and probe, $1 \mu 1$ TBP-specific primers and probe, 100-500 ng of template RNA, and nuclease-free water in a final volume of 20 $\mu 1$. The amplification conditions were as follows: one cycle of $10 \mathrm{~min}$ at $55^{\circ} \mathrm{C}$ and one cycle of $3 \mathrm{~min}$ at $95^{\circ} \mathrm{C}$ followed by 40 cycles of $15 \mathrm{~s}$ at $95^{\circ} \mathrm{C}$ and $30 \mathrm{~s}$ at $60^{\circ} \mathrm{C}$. Fluorescence was detected at the end of the $60{ }^{\circ} \mathrm{C}$ extension step. Each sample was quantified in triplicate, and a 
no-template control (NTC) reaction, with just nuclease-free water, was included as a negative control. The fold difference in CFTR gene expression normalized to TBP was calculated using the comparative $\mathrm{Ct}$ method, $2^{-\Delta \Delta \mathrm{Ct}}$. The following target-specific primers and probe used in RTqPCR experiments were purchased from Integrated DNA technologies (Coralville, IA, USA): CFTR probe, CFTR primer 1, and CFTR primer 2 and endogenous control TBP-specific TBP probe, TBP primer 1, and TBP primer 2 (sequences in Supplementary Table 2). The final concentration of primers was $500 \mathrm{nM}$ each and probe was $250 \mathrm{nM}$.

\section{Ussing chamber studies of $\mathrm{HBE}$ cells}

HBE cells were seeded onto $6.5 \mathrm{~mm}$ Transwell Permeable Support with $0.4 \mu \mathrm{m}$ Polycarbonate Membrane Inserts (Corning, \#3413) pre-coated with Collagen from rat tail (Sigma-Aldrich, \#C7661-25MG) 5-6 days before assay and maintained in normal cell culture media at $37{ }^{\circ} \mathrm{C}$ and $5 \% \mathrm{CO}_{2}$. Cells were grown until the formation of electrically tight epithelial monolayer was confirmed by measuring transepithelial resistance with EVOM $^{2}$ Epithelial Voltohmmeter (World Precision Instruments, Sarasota, FL, USA). Short circuit current $\left(I_{\mathrm{sc}}\right)$ was measured under voltage clamp condition to determine CFTR channel function. Cell monolayers were mounted in Ussing chambers (Physiologic Instruments, San Diego, CA, USA) filled with Ringer's solution (in mM: $135 \mathrm{NaCl}, 5 \mathrm{HEPES}, 0.6 \mathrm{KH}_{2} \mathrm{PO}_{4}, 2.4 \mathrm{~K}_{2} \mathrm{HPO}_{4}, 1.2 \mathrm{MgCl}_{2}, 1.2 \mathrm{CaCl}_{2}, 5$ D-Glucose adjusted to $\mathrm{pH} 7.4$ ) at $37^{\circ} \mathrm{C}$ and gassed with compressed air. After baseline recordings, cells were treated from the apical side with $100 \mu \mathrm{M}$ amiloride to block epithelial sodium channels and then with $100 \mu \mathrm{M}$ 4,4'-diisothiocyanostilbene-2,2'-disulfonic acid (DIDS) to block $\mathrm{Ca}^{2+}$ activated chloride channels. Then, the solution in the apical side was replaced with a low $\mathrm{Cl}^{-}$ solution (in mM: $135 \mathrm{Na}$-Gluconate, $5 \mathrm{HEPES}, 0.6 \mathrm{KH}_{2} \mathrm{PO}_{4}, 2.4 \mathrm{~K}_{2} \mathrm{HPO}_{4}, 1.2 \mathrm{MgCl}_{2}, 1.2 \mathrm{CaCl}_{2}$, 
5 D-Glucose adjusted to $\mathrm{pH}$ 7.4). CFTR-mediated $\mathrm{Cl}^{-}$current was measured by adding CFTR activators, $10 \mu \mathrm{M}$ forskolin and $100 \mu \mathrm{M}$ 3-isobutyl-1-methlyxanthine (IBMX), and CFTR inhibitor, $20 \mu \mathrm{M}$ CFTR inhibitor-172 (CFTRInh-172), to the apical side. Data were acquired with Acquire \& Analyze 2.3 software (Physiologic Instruments, San Diego, CA, USA). The maximum change in $\mathrm{I}_{\mathrm{sc}}$ in response to additions of CFTR activators and CFTR inhibitor were quantified as measures of functional $\mathrm{CFTR}^{-} \mathrm{Cl}^{-}$transport activity.

\section{Macroscopic Recordings of CFTR Currents}

Cells were transfected using Lipofectamine ${ }^{\mathrm{TM}}$ LTX Reagent with PLUS ${ }^{\mathrm{TM}}$ Reagent (Invitrogen) with a modified reverse transfection as described above. W1282X-CFTR 16HBEge cells were transfected with either a plasmid containing 4 copies of ACE-tRNA ${ }^{\text {Leu }}$ with mNG or empty plasmid encoding only mNG. Results were compared to WT $16 \mathrm{HBE} 14 \mathrm{o}$ - cells transfected with empty mNG plasmid. After transfection, cells were lifted using Versene (Gibco, \#15040-066), plated on 35mm dishes coated with human fibronectin (as previously described) at low density, and cells in isolation were patched 2-3 days post transfection. Cells were continuously perfused with external solution (in mM: 145 TEA-Cl, $10 \mathrm{CaCl}_{2}, 10 \mathrm{HEPES}$ adjusted to $\mathrm{pH} 7.4$ with TEA$\mathrm{OH}$ ) supplemented with $10 \mathrm{uM}$ Forskolin (Sigma) and $100 \mathrm{uM} \mathrm{IBMX} \mathrm{(Sigma)} \mathrm{for} \mathrm{maximal}$ activation of CFTRs; dishes of cells were incubated in this solution for at least 5 minutes prior to recording. Green cells were patched using thick-walled borosilicate glass pipettes (Sutter, Novato, CA, USA) pulled to approximately $7 \pm 3$ MOhm using a vertical puller (PC-100, Narishige, Amityville, NY, USA). Pipettes were filled with internal solution (in mM: $30 \mathrm{CsCl}$, 110 Cs-aspartate, $2.5 \mathrm{CaCl}_{2}, 2.5 \mathrm{MgCl}_{2}, 10 \mathrm{HEPES}, 5$ CsEGTA adjusted to $\mathrm{pH} 7.4$ with $\mathrm{CsOH}$ ). Internal and external solutions were formulated to eliminate non-chloride contaminating currents 
(i.e., TEA and cesium to block potassium currents and no sodium present to eliminate sodium currents) and feature a reversal potential for chloride of approximately $-36 \mathrm{mV}$. Currents were amplified using an Axon 200B amplifier (Axon Instruments, Hamden, CT, USA), sampled at 10 $\mathrm{kHz}$ and filtered at $2 \mathrm{kHz}$ using a Digidata 1440A, and recorded using pClamp 10 (Molecular Devices, San Jose, CA, USA). Whole-cell capacitance and series resistance were compensated (approximately $70 \%$ ) and a linear ramp voltage protocol from $-80 \mathrm{mV}$ to $+80 \mathrm{mV}$ (200ms) was then applied from the holding potential $(-40 \mathrm{mV})$ to collect current traces at $0.5 \mathrm{~Hz}$ for 60 sweeps. Perfused solutions were then rapidly exchanged using a fast-step perfusion barrel (VC-8, Warner Instruments) to an external solution containing $20 \mu \mathrm{M}$ CFTR $_{\text {Inh-172 }} 17$ to block CFTR currents and the ramp voltage protocol was continued at $0.5 \mathrm{~Hz}$ for 180 more sweeps. Recordings were analyzed using Clampfit 10 , whereby peak outward current near $+80 \mathrm{mV}$ was divided by cell capacitance, as determined by the membrane test function in pClamp, to yield current densities (pA/pF). Peak outward current density at $+80 \mathrm{mV}$ was then plotted versus time using Prism 9

(Graphpad) for comparison of treatment groups and controls. Only experiments where a gigaohm seal was achieved before break-in and had a measured reversal potential that fell within $7 \mathrm{mV}$ of $-36 \mathrm{mV}$ were used in analysis.

\section{Quantification of stably integrated copy number using quantitative real-time PCR}

Genomic ACE-tRNA integration of the cell population was quantified by performing quantitative real-time PCR (qPCR). Genomic DNA (gDNA) was extracted using the Monarch Genomic DNA Purification Kit (New England BioLabs, \#T3010S) according to manufacturer's instructions. Genomic integrants of the PB transposon expressing 16 copies of ACE-tRNA ${ }^{\mathrm{Arg}}$ cassette (PB 16x ACE-tRNA ${ }^{\mathrm{Arg}}$ ) were detected by targeting either the puromycin cassette (puro) 
or PB backbone (PB backbone) from the PB transposon vector. Puro-specific puro probe, puro primer 1, and puro primer 2 and PB backbone specific PB backbone probe, PB backbone primer 1, and PB backbone primer 2 were purchased from Integrated DNA technologies (Coralville, IA, USA). The gDNA content was normalized based on the amplification of the Ribonuclease P (RNase $P$ ) gene using RNase $P$ probe purchased from Applied Biosystems and RNase P primer 1 and $R$ Nase $P$ primer 2 purchased from Invitrogen. The final concentration of primers was 500 $\mathrm{nM}$ each and probe was $250 \mathrm{nM}$. Real-time PCR was performed on the QuantStudio 3 Real-Time PCR System using the PrimeTime Gene Expression Master Mix (Integrated DNA Technologies, \#1055772) according to manufacturer's guidelines and then analyzed with QuantStudio Design \& Analysis Software v1.5.1. Briefly, the reaction mixture contained $10 \mu 12 x$ PrimeTime Gene Expression Master Mix, 1 l 20x puro- or PB backbone-specific primers and probe (target gene), $1 \mu \mathrm{l}$ RNase $P$-specific primers and probe (reference gene), DNA template and nuclease-free water in a final volume of $20 \mu \mathrm{l}$. PCR cycling conditions were one cycle of $3 \mathrm{~min}$ at $95^{\circ} \mathrm{C}$ followed by 40 cycles of $15 \mathrm{~s}$ denaturation at $95^{\circ} \mathrm{C}$ and 1 min annealing and extension at $60^{\circ} \mathrm{C}$. A standard curve was constructed by serial dilutions of a PB 16x ACE-tRNA ${ }^{\text {Arg }}$ plasmid with puroor PB backbone-specific primers and probe and Hs_RPP30 Positive Control (Integrated DNA technologies, \#10006622) with RNase P-specific primers and probe. The number of ACEtRNA $^{\text {Arg }}$ copies in $10 \mathrm{ng}$ or $1 \mathrm{ng}$ DNA from cells stably integrated with ACE-tRNA ${ }^{\text {Arg }}$ was calculated by plotting cycle threshold $\left(\mathrm{C}_{\mathrm{t}}\right)$ to the linear equation from the standard curve. All qPCR reactions were run in triplicate. A NTC reaction with just nuclease-free water was included as a negative control, and $10 \mathrm{ng}$ or 1ng DNA from cells not integrated with ACEtRNA $^{\text {Arg }}$ was assayed to confirm specificity of puro- and PB backbone-specific primers and probe. 


\section{Statistical analysis}

Data are presented as mean \pm standard error of the mean (SEM). GraphPad Prism 7 software

(GraphPad Software, San Diego, CA, USA) was used for all statistical analysis. Comparisons

between two groups were performed using an unpaired Student's t-test. Multiple comparisons

were analyzed using a one-way analysis of variance (ANOVA) followed by Tukey's multiple

comparisons test. $p$ values $<0.05$ were considered statistically significant.

\section{REFERENCES AND NOTES}

1. Kurosaki T, Popp MW, Maquat LE. Quality and quantity control of gene expression by nonsense-mediated mRNA decay. Nat Rev Mol Cell Biol. 2019;20(7):406-20.

2. Mort M, Ivanov D, Cooper DN, Chuzhanova NA. A meta-analysis of nonsense mutations causing human genetic disease. Hum Mutat. 2008;29(8):1037-47.

3. Bladen CL, Salgado D, Monges S, Foncuberta ME, Kekou K, Kosma K, et al. The TREAT-NMD DMD Global Database: analysis of more than 7,000 Duchenne muscular dystrophy mutations. Hum Mutat. 2015;36(4):395-402.

4. Lefebvre S, Bürglen L, Reboullet S, Clermont O, Burlet P, Viollet L, et al. Identification and characterization of a spinal muscular atrophy-determining gene. Cell. 1995;80(1):155-65.

5. Chang JC, Kan YW. beta 0 thalassemia, a nonsense mutation in man. Proc Natl Acad Sci U S A. 1979;76(6):2886-9.

6. Ballabio A, Gieselmann V. Lysosomal disorders: from storage to cellular damage. Biochim Biophys Acta. 2009;1793(4):684-96.

7. Reiners J, Nagel-Wolfrum K, Jürgens K, Märker T, Wolfrum U. Molecular basis of human Usher syndrome: deciphering the meshes of the Usher protein network provides insights into the pathomechanisms of the Usher disease. Exp Eye Res. 2006;83(1):97-119.

8. Cheng SH, Gregory RJ, Marshall J, Paul S, Souza DW, White GA, et al. Defective intracellular transport and processing of CFTR is the molecular basis of most cystic fibrosis. Cell. 1990;63(4):827-34.

9. Kerem B, Rommens JM, Buchanan JA, Markiewicz D, Cox TK, Chakravarti A, et al. Identification of the cystic fibrosis gene: genetic analysis. Science. 1989;245(4922):1073-80.

10. Riordan JR, Rommens JM, Kerem B, Alon N, Rozmahel R, Grzelczak Z, et al. Identification of the cystic fibrosis gene: cloning and characterization of complementary DNA. Science. 1989;245(4922):1066-73.

11. Rommens JM, Zengerling-Lentes S, Kerem B, Melmer G, Buchwald M, Tsui LC. Physical localization of two DNA markers closely linked to the cystic fibrosis locus by pulsed-field gel electrophoresis. Am J Hum Genet. $1989 ; 45(6): 932-41$. 
12. The Clinical and Functional TRanslation of CFTR (CFTR2) [Available from: http://cftr2.org.

13. Condren ME, Bradshaw MD. Ivacaftor: a novel gene-based therapeutic approach for cystic fibrosis. J

Pediatr Pharmacol Ther. 2013;18(1):8-13.

14. Van Goor F, Hadida S, Grootenhuis PD, Burton B, Stack JH, Straley KS, et al. Correction of the F508delCFTR protein processing defect in vitro by the investigational drug VX-809. Proc Natl Acad Sci U S A.

2011;108(46):18843-8.

15. Sala MA, Jain M. Tezacaftor for the treatment of cystic fibrosis. Expert Rev Respir Med. 2018;12(9):725-

32.

16. Rommens JM, Iannuzzi MC, Kerem B, Drumm ML, Melmer G, Dean M, et al. Identification of the cystic fibrosis gene: chromosome walking and jumping. Science. 1989;245(4922):1059-65.

17. Drumm ML, Pope HA, Cliff WH, Rommens JM, Marvin SA, Tsui LC, et al. Correction of the cystic fibrosis defect in vitro by retrovirus-mediated gene transfer. Cell. 1990;62(6):1227-33.

18. Cooney AL, McCray PB, Jr., Sinn PL. Cystic Fibrosis Gene Therapy: Looking Back, Looking Forward. Genes (Basel). 2018;9(11).

19. Rees HA, Liu DR. Base editing: precision chemistry on the genome and transcriptome of living cells. Nat Rev Genet. 2018;19(12):770-88.

20. Min YL, Bassel-Duby R, Olson EN. CRISPR Correction of Duchenne Muscular Dystrophy. Annu Rev Med. 2019;70:239-55.

21. Krishnamurthy S, Wohlford-Lenane C, Kandimalla S, Sartre G, Meyerholz DK, Theberge V, et al. Engineered amphiphilic peptides enable delivery of proteins and CRISPR-associated nucleases to airway epithelia. Nat Commun. 2019;10(1):4906.

22. Bedwell DM, Kaenjak A, Benos DJ, Bebok Z, Bubien JK, Hong J, et al. Suppression of a CFTR premature stop mutation in a bronchial epithelial cell line. Nat Med. 1997;3(11):1280-4.

23. Howard M, Frizzell RA, Bedwell DM. Aminoglycoside antibiotics restore CFTR function by overcoming premature stop mutations. Nat Med. 1996;2(4):467-9.

24. Keeling KM, Xue X, Gunn G, Bedwell DM. Therapeutics based on stop codon readthrough. Annu Rev Genomics Hum Genet. 2014;15:371-94.

25. Dabrowski M, Bukowy-Bieryllo Z, Zietkiewicz E. Advances in therapeutic use of a drug-stimulated translational readthrough of premature termination codons. Mol Med. 2018;24(1):25.

26. Wangen JR, Green R. Stop codon context influences genome-wide stimulation of termination codon readthrough by aminoglycosides. Elife. 2020;9.

27. Temple GF, Dozy AM, Roy KL, Kan YW. Construction of a functional human suppressor tRNA gene: an approach to gene therapy for beta-thalassaemia. Nature. 1982;296(5857):537-40.

28. Panchal RG, Wang S, McDermott J, Link CJ, Jr. Partial functional correction of xeroderma pigmentosum group A cells by suppressor tRNA. Hum Gene Ther. 1999;10(13):2209-19.

29. Bordeira-Carrico R, Ferreira D, Mateus DD, Pinheiro H, Pego AP, Santos MA, et al. Rescue of wild-type E-cadherin expression from nonsense-mutated cancer cells by a suppressor-tRNA. Eur J Hum Genet.

2014;22(9):1085-92.

30. Lueck JD, Yoon JS, Perales-Puchalt A, Mackey AL, Infield DT, Behlke MA, et al. Engineered transfer RNAs for suppression of premature termination codons. Nat Commun. 2019;10(1):822.

31. Porter JJ, Heil CS, Lueck JD. Therapeutic promise of engineered nonsense suppressor tRNAs. Wiley Interdiscip Rev RNA. 2021;12(4):e1641.

32. Valley HC, Bukis KM, Bell A, Cheng Y, Wong E, Jordan NJ, et al. Isogenic cell models of cystic fibrosiscausing variants in natively expressing pulmonary epithelial cells. J Cyst Fibros. 2019;18(4):476-83.

33. Dorin JR, Farley R, Webb S, Smith SN, Farini E, Delaney SJ, et al. A demonstration using mouse models that successful gene therapy for cystic fibrosis requires only partial gene correction. Gene Ther. 1996;3(9):797-801. 34. Kerem E. Pharmacologic therapy for stop mutations: how much CFTR activity is enough? Curr Opin Pulm Med. 2004;10(6):547-52.

35. Burnight ER, Staber JM, Korsakov P, Li X, Brett BT, Scheetz TE, et al. A Hyperactive Transposase Promotes Persistent Gene Transfer of a piggyBac DNA Transposon. Mol Ther Nucleic Acids. 2012;1:e50.

36. Hall MP, Unch J, Binkowski BF, Valley MP, Butler BL, Wood MG, et al. Engineered luciferase reporter from a deep sea shrimp utilizing a novel imidazopyrazinone substrate. ACS Chem Biol. 2012;7(11):1848-57.

37. Xue X, Mutyam V, Thakerar A, Mobley J, Bridges RJ, Rowe SM, et al. Identification of the amino acids inserted during suppression of CFTR nonsense mutations and determination of their functional consequences. Hum Mol Genet. 2017;26(16):3116-29. 
38. Middleton T, Sugden B. Retention of plasmid DNA in mammalian cells is enhanced by binding of the Epstein-Barr virus replication protein EBNA1. J Virol. 1994;68(6):4067-71.

39. Chen ZY, He CY, Meuse L, Kay MA. Silencing of episomal transgene expression by plasmid bacterial DNA elements in vivo. Gene Ther. 2004;11(10):856-64.

40. Buvoli M, Buvoli A, Leinwand LA. Suppression of nonsense mutations in cell culture and mice by multimerized suppressor tRNA genes. Mol Cell Biol. 2000;20(9):3116-24.

41. Wang W, Lin C, Lu D, Ning Z, Cox T, Melvin D, et al. Chromosomal transposition of PiggyBac in mouse embryonic stem cells. Proc Natl Acad Sci U S A. 2008;105(27):9290-5.

42. Yusa K, Rad R, Takeda J, Bradley A. Generation of transgene-free induced pluripotent mouse stem cells by the piggyBac transposon. Nat Methods. 2009;6(5):363-9.

43. Kolacsek O, Krizsik V, Schamberger A, Erdei Z, Apati A, Varady G, et al. Reliable transgene-independent method for determining Sleeping Beauty transposon copy numbers. Mob DNA. 2011;2(1):5.

44. Shoemaker CJ, Green R. Translation drives mRNA quality control. Nat Struct Mol Biol. 2012;19(6):594-

601.

45. Chen Y, Ma J, Lu W, Tian M, Thauvin M, Yuan C, et al. Heritable expansion of the genetic code in mouse and zebrafish. Cell Res. 2017;27(2):294-7.

46. Shi N, Yang Q, Zhang H, Lu J, Lin H, Yang X, et al. Restoration of dystrophin expression in mice by suppressing a nonsense mutation through the incorporation of unnatural amino acids. Nat Biomed Eng. 2021.

47. Kowalski PS, Rudra A, Miao L, Anderson DG. Delivering the Messenger: Advances in Technologies for Therapeutic mRNA Delivery. Mol Ther. 2019;27(4):710-28.

48. Hornstein BD, Roman D, Arévalo-Soliz LM, Engevik MA, Zechiedrich L. Effects of Circular DNA Length on Transfection Efficiency by Electroporation into HeLa Cells. PLoS One. 2016;11(12):e0167537.

49. Lukacs GL, Haggie P, Seksek O, Lechardeur D, Freedman N, Verkman AS. Size-dependent DNA mobility in cytoplasm and nucleus. J Biol Chem. 2000;275(3):1625-9.

50. Mieruszynski S, Digman MA, Gratton E, Jones MR. Characterization of exogenous DNA mobility in live cells through fluctuation correlation spectroscopy. Sci Rep. 2015;5:13848.

51. Dean DA. Nonviral gene transfer to skeletal, smooth, and cardiac muscle in living animals. Am J Physiol Cell Physiol. 2005;289(2):C233-45.

52. Ramamoorth M, Narvekar A. Non viral vectors in gene therapy- an overview. J Clin Diagn Res. 2015;9(1):GE01-6.

53. Shen H, Huang X, Min J, Le S, Wang Q, Wang X, et al. Nanoparticle Delivery Systems for DNA/RNA and their Potential Applications in Nanomedicine. Curr Top Med Chem. 2019;19(27):2507-23.

54. Bazzani RP, Pringle IA, Connolly MM, Davies LA, Sumner-Jones SG, Schleef M, et al. Transgene sequences free of $\mathrm{CG}$ dinucleotides lead to high level, long-term expression in the lung independent of plasmid backbone design. Biomaterials. 2016;93:20-6.

55. Gill DR, Smyth SE, Goddard CA, Pringle IA, Higgins CF, Colledge WH, et al. Increased persistence of lung gene expression using plasmids containing the ubiquitin $\mathrm{C}$ or elongation factor 1alpha promoter. Gene Ther. 2001;8(20):1539-46.

56. Dean DA. Electroporation of the vasculature and the lung. DNA Cell Biol. 2003;22(12):797-806.

57. Lundstrom K. Viral Vectors in Gene Therapy. Diseases. 2018;6(2).

58. Yusa K, Zhou L, Li MA, Bradley A, Craig NL. A hyperactive piggyBac transposase for mammalian applications. Proc Natl Acad Sci U S A. 2011;108(4):1531-6.

59. Cozens AL, Yezzi MJ, Kunzelmann K, Ohrui T, Chin L, Eng K, et al. CFTR expression and chloride secretion in polarized immortal human bronchial epithelial cells. Am J Respir Cell Mol Biol. 1994;10(1):38-47.

\section{Acknowledgments}

We thank members of the Lueck Laboratory and Dr. Amy M. Martin for reading and editing the manuscript and constructive discussion throughout the study. We thank T.N. Lueck with help 
Dr. Hillary Valley for providing 16HBE14o- and 16HBE14ge cell lines used in this study and Dr. David MacLean for extended use of his electrophysiology hardware.

\section{Funding}

This work was funded by the Cystic Fibrosis Foundation Postdoctoral Fellowship

(PORTER20F0) to J.J.P and a Cystic Fibrosis Foundation Research Grant (LUECK18GO),

Vertex Pharmaceutical Cystic Fibrosis Research Innovation Award and NIH grant (1 R01

HL153988-01A1) to J.D.L.

\section{Author Contributions}

J.J.P., W.K., and J.D.L designed the study. J.J.P., W.K., M.T.S, K.M.E and J.D.L. performed experiments. J.J.P., W.K., M.T.S, and J.D.L. analyzed the data, and constructed the figures. J.J.P, W.K., and J.D.L. wrote the manuscript. All authors read and revised the manuscript.

\section{Conflict of Interest Statement}

John D. Lueck is a co-inventor of the technology presented in this study and receives royalty payments related to the licensing of the technology from the University of Iowa.

\section{Data and Material Availability}

All data are available in the main text or the supplementary materials. 